\title{
Fast ultrasonic phased array inspection of complex geometries delivered through robotic manipulators and high speed data acquisition instrumentation
}

\author{
Carmelo Mineo, Charles MacLeod, Maxim Morozov, \\ S. Gareth Pierce, Timothy Lardner, Rahul Summan \\ Dept. of Electronic and Electrical Eng., University of \\ Strathclyde, Glasgow, United Kingdom \\ carmelo.mineo@strath.ac.uk
}

\author{
Jonathan Powell, Paul McCubbin, Coreen McCubbin, \\ Gavin Munro, Scott Paton and David Watson \\ Spirit AeroSystems (Europe) Limited \\ Prestwick, United Kingdom
}

David Lines

Diagnostic Sonar Ltd, Livingston, United Kingdom.

\begin{abstract}
Performance of modern robotic manipulators has enabled research and development of fast automated nondestructive testing (NDT) systems for complex geometries. This paper presents recent outcomes of work aimed at removing the bottleneck due to data acquisition rates, to fully exploit the scanning speed of modern 6-DoF manipulators. State of the art ultrasonic instrumentation has been integrated into a large robot cell to enable fast data acquisition, high scan resolutions and accurate positional encoding. A fibre optic connection between the ultrasonic instrument and the server computer enables data transfer rates up to $1.6 \mathrm{~GB} / \mathrm{s}$. Multiple data collection methods are compared. Performance of the integrated system allows traditional ultrasonic phased array scanning as well as full matrix capture (FMC). In FMC configuration, linear scan speeds up to $156 \mathrm{~mm} / \mathrm{s}$ with 64 pulses per frame are achieved - this speed is only constrained by the acoustic wave propagation in the component. An $8 \mathrm{x}$ increase of the speed (up to $1.25 \mathrm{~m} / \mathrm{s}$ ) can be achieved using multiple transmission elements, reaching the physical limits for acceptable acoustic alignment of transmission and reception paths. Scan results, relative to a $1.2 \mathrm{~m} \times 3 \mathrm{~m}$ carbon fibre sample, are presented.
\end{abstract}

\section{Keywords-Phased array; FMC; Robotic NDT; CFRP}

\section{INTRODUCTION}

The aerospace industry faces, more than other sectors, the need to speed up the inspection of critical components. To cope with future demand projections for modern aircrafts, it is essential to overcome the current NDT bottlenecks. Manual scanning is a slow process for large samples and requires trained technicians. Moreover, it can be challenging to obtain repeatable results in structures where complex inspection setups are required [1]. Developing reliable automated solutions has become an industrial priority to speed up repetitive inspections in the production chain.

Semi-automated inspection systems have been developed to overcome some of the shortcomings of the manual inspection techniques, using both mobile and fixed robotic platforms. For a

This work was developed through the VIEWS project (Validation and Integration of Manufacturing Enablers for Future Wing Structures), part funded by the UK's innovation agency (Innovate UK) and Spirit AeroSystems (Prestwick, UK) number of years, the use of linear manipulators and bridge designs has provided the most stable conditions in terms of positioning accuracy [2]. The use of these systems to inspect parts with non-complex shapes (plates, cylinders or cones) is widespread. Typically, they are specific machines which are used to inspect identically shaped and/or sized parts. In the spectrum of robot manipulators, some modern robots have suitable attributes to develop automated NDT systems and cope with the challenging requirements posed by the aerospace industry [3]. They include precise mechanics and the ability to export positional data at frequencies up to $1 \mathrm{kHz}$. Some applications of 6-axis robotic arms in the NDT field have been developed and published during the last few years, resulting in a growing interest in using such automation solutions [3-6].

Despite these previous efforts, there remain challenges to be addressed. Current research is tackling key challenges: the generation of flexible and adaptive robot tool-paths, the development of high speed NDT data collection and the integration of surface metrology measurements. New data visualization and fast data analysis methods are required to truly speed up the overall throughput of the inspection process.

Traditionally, NDE and metrology measurements are undertaken at different stages of a product manufacture cycle using specific dedicated equipment and personnel. However, since both processes involve direct interaction with the component's surface, motivations exist to combine NDE and metrology to potentially reduce overall cycle time. The VIEWS project (Validation and Integration of Manufacturing Enablers for Future Wing Structures), part funded by the UK's innovation agency (Innovate UK), is promoting collaboration between the University of Strathclyde and Spirit AeroSystems. This collaboration is establishing an automated hybrid cell demonstrator, which is capable of both ultrasonic NDT and metrology inspection. This paper focuses on the NDT development carried out within the project. Emphasis is given to the ultrasonic (UT) instrumentation and the way it has been interfaced to the robotics. 


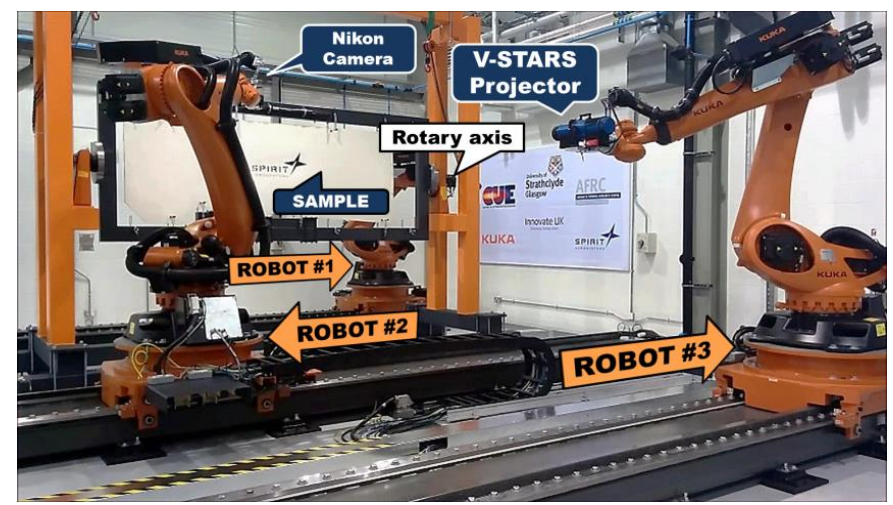

Fig. 1. VIEWS multi-robot flexible inspection at the University of Strathclyde

\section{SYSTEM DESCRIPTION}

\section{A. Robotics}

The VIEWS robotic inspection cell is shown in Fig. 1. It comprises three industrial robots, mounted on linear tracks. A custom tooling frame was purposely designed to support an external axis drive unit utilized for work-piece manipulation. The cell occupies an area of $103 \mathrm{~m}^{2}$, surrounded by fixed safety guarding with access gates. Labels are superposed to the photo in Fig. 1 to indicate the robots, the external rotary axis, the target sample and the metrology instrumentation. The controller of Robot \#1 controls the additional external axis drive unit, utilized to enable rotation of the rigid frame where the part is secured. The frame is supported, through the drive unit and the opposite tailstock, by a towering static frame.

\section{B. Ultrasonic transducer}

Traditional methods to perform UT scans of components require the use of a single-element probe or a multiplexed group of probes. Ultrasonic single-element (SE) and phased array (PA) testing have been integrated into several robotic inspection system prototypes over recent years [6-8]. Since the ultrasonic probes require coupling with the surface of the part under inspection, these prototypes have used purposely developed water jet nozzles that embed the transducer and provide a water column for the transmission of the ultrasonic beam.

This work enables robotic ultrasound inspection by means of an ultrasonic array probe housed within a rubber coupled wheel sensor. This approach was originally developed for faster manual inspection techniques of large components. It removes the need for a free water column. The RollerFORM® phased array wheel probe by Olympus is used. It embeds a $5 \mathrm{MHz}, 64-$ element, $0.8 \mathrm{~mm}$ pitch transducer. The wheel probe comes with an ergonomically designed handle to allow manual inspection, as it is shown in Fig. 2a. In our integration, the wheel probe was removed from the handle and mounted onto Robot \#1, through a purposely designed support (see Fig. 2b).

The RollerFORM imposes limitations on the tolerable standoff variations between the probe and the part surface. Therefore, Robot \#1 was equipped with a Force Torque (FT) sensor, mounted between the robot flange and the wheel probe support (Fig. 2b).

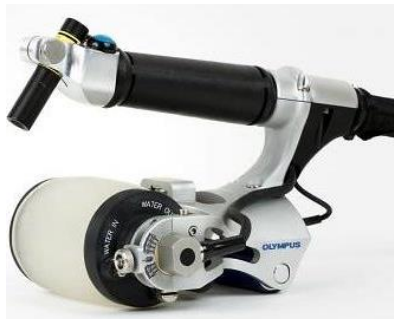

(a)

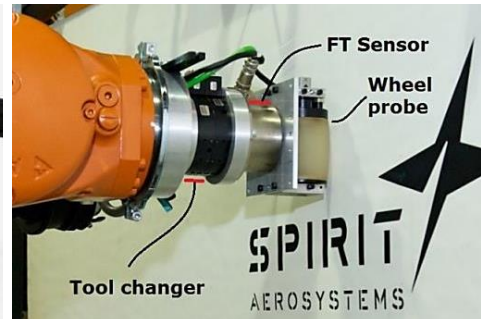

(b)
Fig. 2. RollerFORM® in its original configuration (a) and mounted onto the robot through a support (b)

\section{Data acquisition instrument}

The typical portable ultrasonic instruments are designed for delivery of manual scanning and their limited memory is not suitable to collect large high-resolution C-scan images, which is essential for the automated inspection of large aerospace components. Moreover, the data acquisition rate of such devices is a bottleneck that would limit the scanning speed to values well below the maximum speed of industrial 6-DoF manipulators. Additionally, portable instruments have limited features for data encoding and only 2D representations of the results are typically available.

A customized instrument was designed, commissioned and procured through Diagnostic Sonar Ltd (DSL). This is herein referred as FIToolbox instrument. The instrument enables high data rates by exploiting the flexibility of Field Programmable Gate Arrays (FPGAs) in a standard PC platform [9]. The FPGAs can be reconfigured under software control to provide a flexible platform, handling applications from array imaging to multichannel automated inspection systems. The FIToolbox system uses off-the-shelf modules, here configured as 64 channels of high-speed analogue acquisition with high performance FPGAs for real-time, deterministic processing. It is a 64Tx/64Rx system that interfaces to the ultrasonic array probe via multi-channel pulser-receivers with custom FPGA code developed using LabVIEW®. The instrument enables high performance multichannel ultrasound acquisition and processing, while being flexible enough to address a wide range of research applications. FIToolbox is modular and the independent nature of the transmitter and receiver modules means that both pulse-echo and through-transmission modes are supported.

The data transfer rate of high performance phased array receivers is often limited by the connection between the device and the controlling computer. This is not the case for the FIToolbox, where National Instrument MXI-Express hardware enables direct communication with computer memory via the PCI Express bus that provides the highest bandwidth of all PC $\mathrm{I} / \mathrm{O}$ buses. The $50 \mathrm{~m}$ fibre optic cable option allows data streaming at up to $1.6 \mathrm{~GB} / \mathrm{s}$ into the PC. The instrument was mounted the carriage of Robot \#1 within a protective splash proof cover that provides Ingress Protection (IP) rate 53.

\section{Integration}

In order to enable the high level of automation required by the VIEWS project objectives, fundamental software was developed to establish robust communications between the robot controllers, the metrology equipment, the NDT instrumentation and the server computer. $\mathrm{C} \#$ and $\mathrm{C}++$ programming language 
were chosen to develop crucial software components. These languages allow a low level control of the communication strategies and are suitable to develop data acquisition algorithms that run in a reliable manner. The crucial modules were compiled in Dynamic Link Libraries (DLL). The robotic DLL, integrated in the data acquisition FIToolbox LabVIEW $^{\circledR}$ platform, enables the use of the robot positional feedback to encode the ultrasound data.

\section{OPTIONS FOR HIGH SPEED IMAGING}

The FIToolbox data collection software platform contains several modules. The robotic control software was integrated with the FIToolbox's Full Raw Data (FRD) streaming module, which was developed by DSL to allow acquisition of FRD datasets. FRD allows the option of sparse data collection as well as variable numbers of transmit channels in each acquisition with differential delays. It is the most versatile way to acquire data from ultrasonic array probes. Full Matrix Capture (FMC) is a special case of FRD, where the matrix of transmitting and receiving elements has to be fully populated and only one transmitter is fired at a time. FMC is the most demanding method, for the required transfer speed.

With pulse repetition frequency (PRF) of $10 \mathrm{kHz}$ and a $64-\mathrm{el}$. probe, the theoretical rate of frames per second (FPS) is $156 \mathrm{~Hz}$ (10kHz/64).

Consider the acquisition of a $26 \mu$ s waveform per each element, at a sampling frequency of $50 \mathrm{MHz}$, giving 1300 samples per beam. For 64 FMC channels at 2 Bytes per sample, the frame size $(f s)$ is $f s=1300 \cdot 64^{2} \cdot 2$ Bytes $=10.16 \mathrm{MB}$. Therefore, the data transfer rate $(t r)$ is $1.55 \mathrm{~GB} / \mathrm{s}(f s \cdot \mathrm{FPS})$, which is continuously sustainable $(<1.6 \mathrm{~GB} / \mathrm{s})$. Despite the transfer rate being sustainable, data acquired as FMC impose a constraining limitation on the robot scanning speed, if compared to the robot rated speed limit of $2 \mathrm{~m} / \mathrm{s}$. The desired resolution in the scanning direction $(r)$ limits the scan speed (s). For a resolution of $1 \mathrm{~mm}, s=r \cdot F P S=156 \mathrm{~mm} / \mathrm{s}$.

Acquiring the data and beamforming inside the FPGA does not give significant frame rate increase as it is limited by PRF (fixed by transit time) and number of lines. A 64-el. probe with a transmitting/receiving sub-aperture of 8 elements would produce 57 lines/frame, producing a mere $12 \%$ increase in the maximum scanning speed $(64 / 57=1.12)$. Two data acquisition strategies have been identified for enabling high speed ultrasonic imaging, herein referred as Paintbrush FRD and Multitransmission beam acquisition and beam-forming .

\section{A. Paintbrush FRD}

The method is based on the use of FRD acquisition with transmission aperture set to the full array. This means only 1 pulse per frame is required, resulting in the frame rate being equal to the PRF (10kHz), with no data rate change as still 64 channels/pulse are collected. The method allows a theoretical scanning speed equal to $s=r \cdot P R F$. Maintaining $r=1 \mathrm{~mm}$, $s=10 \mathrm{~m} / \mathrm{s}$. However, all elements fired at once generate a wide flat wave front and there is no focusing on the beam profile.

\section{B. Multi-transmission beam acquisition and beam-forming}

For the 64-el. array embedded in the RollerFORM probe (operating with 8-element aperture to match the aperture width to the probe elevation of $6.4 \mathrm{~mm}$ ), the basic idea consists in enabling the acquisition of a complete frame to produce 57 beams from just 8 pulses. This can be done through parallel firing of multi-transmission sub-apertures along the array, possibility enabled by the FIToolbox hardware in use. There is no change in the required acquisition data rate, as still 64 channels/pulse would be acquired.

This method gives an 8x speedup. The multi-transmission beam acquisition allows exploiting the FPGAs for beamforming. The 8 pulses per frame at $10 \mathrm{kHz}$ PRF produces a frame acquisition time of $800 \mu \mathrm{s}$. The maximum robot scanning speed is $1.25 \mathrm{~m} / \mathrm{s}$ and the 8 transmissions are offset by $125 \mu \mathrm{m}$ in the scan direction on the reconstructed C-scan. This should still be acceptable for acoustic alignment of Tx and Rx paths.

One advantage of the 8-element reconstruction aperture is that it is an integer sub-multiple of the number of channels per card. This means that a low cost FIToolbox hardware version can be developed with a multiplexed single transmission card and multiple parallel cards for receiving and digitizing.

\section{RESULTS}

\section{A. Quantitative performance comparison}

A commercial scanner performing SE pulse-echo inspection and a system performing PA pulse-echo inspection, both based on 3-DoF gantry probe manipulation, were tested. The first system was capable of reaching a scanning rate of $3.6 \mathrm{~m}^{2} /$ hour (assuming a $1 \mathrm{~mm}$ step between parallel raster scan passes). The second system benefitted from the wide PA probe active area, showing scanning rate of $8.1 \mathrm{~m}^{2} /$ hour.

Table 1 compares the acquisition rate of the 3-DoF scanners with the scanning rates of the VIEWS system. The table does not show the performances for the multi-transmission beam acquisition method described above, since the software implementation of this acquisition strategy is on-going. The initial scan trials were carried out with a simple linear PA sweep of 1-element Tx/Rx aperture across the active axis of the array. The paintbrush FRD approach was also tested.

The VIEWS system shows a scanning rate of $18.1 \mathrm{~m}^{2} /$ hour with the pulse-echo $1 \mathrm{Tx} / 1 \mathrm{Rx}$ approach and an ultrasonically reachable scanning rate of $117.6 \mathrm{~m}^{2} /$ hour with the pulse-echo paintbrush approach. The first method is 2.1 times faster than the PA 3-DoF scanner and 5 times faster than the SE system. The paintbrush method provides a PA scanning system 13.5 times faster than the PA 3-DoF scanner.

TABLE I. SCANNING RATES OF COMMERCIAL 3-DOF SCANNERS AND VIEWS 6-DOF SYSTEM.

\begin{tabular}{|l|c|c|c|c|}
\hline & \multicolumn{2}{|c|}{ 3-DoF scanners } & \multicolumn{2}{c|}{ VIEWS NDT system } \\
\hline Type of inspection & SE & PA & PA & PA \\
\hline Probe & & 64el - 1.0mm & \multicolumn{2}{c|}{ 64el - 0.8mm } \\
\hline Data acquisition strategy & & Beam-forming & 1Tx/1Rx & Paintbrush \\
\hline Sub-aperture & 1 & 10 & 1 & 58 \\
\hline Scanning res. (mm) & 1 & 1 & 1 & 1 \\
\hline Theoretical FPS (Hz) & 10000 & 185.18 & 156.25 & 10000 \\
\hline FPS (Hz) & 1000 & 44 & 100 & 715 \\
\hline Max scan speed (mm/s) & 1000 & 44 & 100 & 715 \\
\hline Scanning step (mm) & 1 & 54.98 & 50.4 & 45.6 \\
\hline Scan rate $\left(\mathrm{m}^{2} / \mathrm{hour}\right)$ & 3.6 & 8.7 & 18.1 & 117.6 \\
\hline
\end{tabular}


(a)

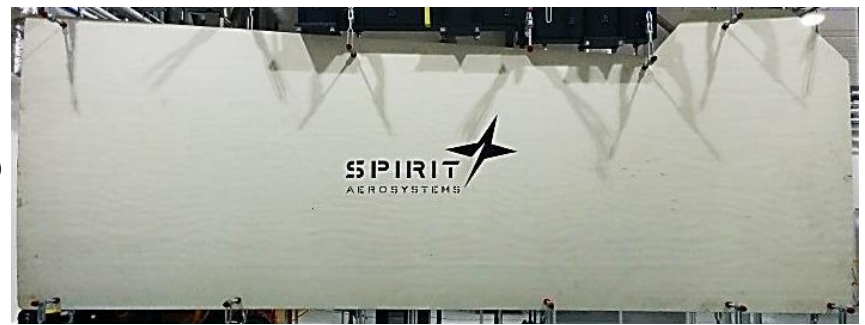

(b)

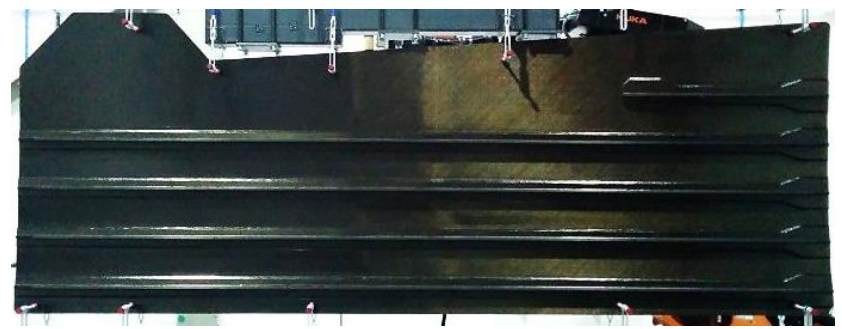

Fig. 3. Sub-scale wing cover: Outer Mould Line (a) and Inner Mould Line (b)

\section{B. C-scans}

Fig. 3 shows a sub-scale wing cover section, measuring approximately $1.2 \mathrm{~m} \times 3 \mathrm{~m}$ and of thickness varying from 6 to $18 \mathrm{~mm}$. It is a carbon fibre part with stringers bonded to the inner mould line. This sample was used to test the robotic inspection.

A full reference manual scan was acquired through the standard configuration of the RollerFORM probe (see Fig. 2a), linked to an OmniScan instrument. To overcome the OmniScan limited operating memory of circa $300 \mathrm{MB}$, the sample surface was partitioned in four regions that were scanned separately. Two calibrated string encoders were used to produce $\mathrm{x}-\mathrm{y}$ coordinates by triangulation. The partitioning of the sample surface allowed achieving a resolution of $1.6 \mathrm{~mm}$ along the phased array probe axis (using a 2-el. step of the PA subapertures) and $2 \mathrm{~mm}$ in the scanning direction. The four subscans were downloaded to a computer and merged through the InspectionWare software, developed by UTEX Scientific Ltd. Fig. 4 shows the resulting merged depth map.

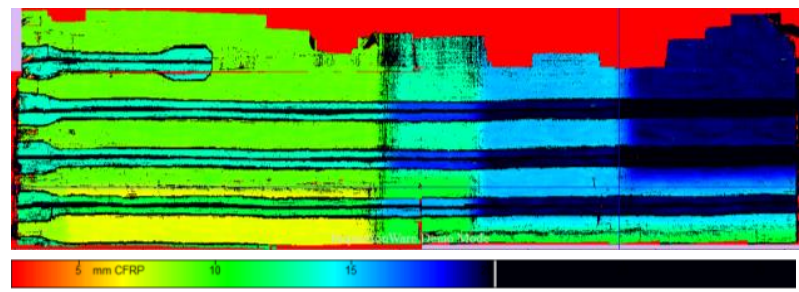

Fig. 4. Merged depth map resulting from manual scan.

The sample was scanned twice through the VIEWS system, firstly using the $1 T x / 1 R x$ method and secondly with the paintbrush method. Higher amount of UT energy penetrates the sample when using the paintbrush approach, since all array elements are triggered at once, producing higher back wall reflections. The robot positional feedback, used to encode the NDT data, produces 3D NDT maps free of misalignments. Fig. 5 shows the top view of the obtained c-scans, both with resolution of $0.8 \mathrm{~mm}$.

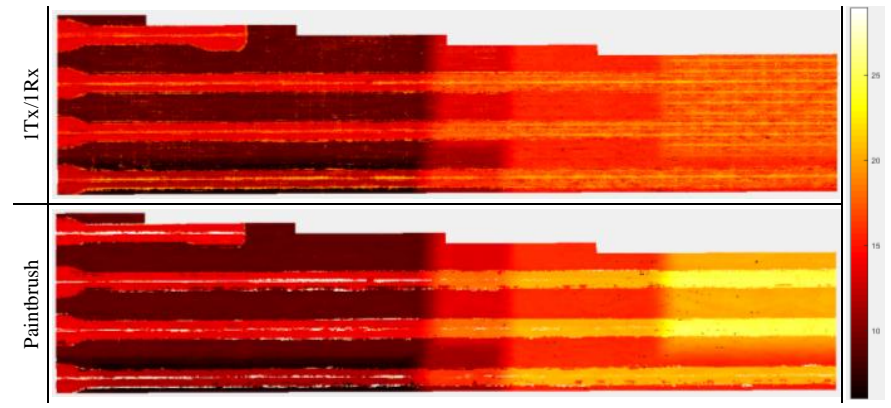

Fig. 5. Depth maps obtained using the VIEWS robotic system.

\section{CONCLUSIONS}

High acquisition rates are required to fully exploit the speed of modern 6-DoF manipulators. State of the art UT instrumentation was integrated into a large robot cell to enable fast data acquisition, high scan resolutions and accurate positional encoding to obtain 3D scan maps. Two data acquisition strategies were identified for enabling high speed UT imaging and exploiting the $1.6 \mathrm{~GB} / \mathrm{s}$ transfer rate, enabled by fibre optic connection between the DSL FIToolbox instrument and the server computer. The robotic inspection system prototype shows, to date, a scanning rate of $18.1 \mathrm{~m}^{2} /$ hour with the $1 \mathrm{Tx} / 1 \mathrm{Rx}$ approach and an ultrasonically reachable scan rate of $117.6 \mathrm{~m}^{2} /$ hour with the paintbrush approach. The robot speed was intentionally limited to $150 \mathrm{~mm} / \mathrm{s}$ for the results described in this paper. Faster robot manipulation requires optimization of the force torque sensor control to cope with the more challenging dynamic constraints given by high speed. This optimization work and the software implementation of the multi-transmission beam-forming acquisition method is currently on-going.

\section{ACKNOWLEDGMENT}

We acknowledge Dr. R. Freemantle (Wavelength NDT Ltd) for acquiring the manual reference scan.

\section{REFERENCES}

[1] T. Sattar, "Robotic non-destructive testing," Industrial Robot: An International Journal, vol. 37, 2010.

[2] P. Louviot, A. Tachattahte, and D. Garnier, "Robotised UT Transmission NDT of Composite Complex Shaped Parts," presented at the 4th International Symposium on NDT in Aerospace, Berlin (Germany), 2012.

[3] E. Cuevas, M. López, and M. García, "Ultrasonic Techniques and Industrial Robots: Natural Evolution of Inspection Systems," presented a the 4th International Symposium on NDT in Aerospace, Berlin (Germany), 2012.

[4] F. Bentouhami, B. Campagne, E. Cuevas, T. Drake, M. Dubois, T. Fraslin, et al., "LUCIE - A flexible and powerful Laser Ultrasonic system for inspection of large CFRP components.," presented at the 2nd International Symposium on Laser Ultrasonics, Talence (France), 2010.

[5] J. T. Stetson and W. D. Odorico, "Robotic inspection of fiber reinforced aerospace composites using phased array UT," presented at the 40th Annual Review of Progress in Quantitative NDE, Baltimore, Maryland, 2013.

[6] C. Mineo, S. Pierce, B. Wright, I. Cooper, and P. Nicholson, "PAUT inspection of complex-shaped composite materials through six DOFs robotic manipulators," Insight-Non-Destructive Testing and Condition Monitoring, vol. 57, pp. 161-166, 2015.

[7] Robotic Non Destructive Inspection NSpect 210. Available: http://genesissystems.com/products/nspect-systems - Accessed 30/6/2016

[8] Tecnatom, "Tecnatom passes the acceptance tests for two new aeronautical systems," NDT.net e-Journal, vol. 19, 2013.

[9] D. Lines, J. Wharrie, and J. Hottenroth, "Multi-channel ultrasound toolbox: A flexible modular approach for real-time array imaging and automated inspection," in ASNT Conference, Houston, 2010, pp. 15-18. 